\title{
OCORRENCIA DE AFLATOXINA EM FARELOS DE AMENDOIM (Arachis hypogaea L.) NA REGIÃO PAULISTA NOVA, DO ESTADO DE SÃO PAULO *
}

\author{
Homero Fonseca
}

\begin{abstract}
RESUMO
No presente trabalho foram estudadas, a ocorrência das aflatoxinas $\mathrm{B}$ e G, em 116 amostras de farelo de amendoim provenientes de 17 fábricas de óleo da região Paulista Nova, bem como uma possivel correlação na produção de ambas.

As amostras foram coletadas em quatro épocas, representando material proveniente da industrialização de duas safras distintas, a saber: março e maio - safra das «águas» e julho e setembro safra da «seca».

Dos resultados concluiu-se que: a) a incidência de aflatoxina foi geral na região, pois todas as amostras estavam tóxicas; b) o nivel de toxidez encontrado foi elevado (valores de 0,1 a $20,0 \mathrm{ppm}$ ) sendo bem mais elevado na safra das «águas» - média de $5,50 \mathrm{ppm}$, contra 1,76 na «seca»; c) apenas $10,35 \%$ do material examinado estaria em condicões de ser utilizado na alimentação animal; d) houve ums correlação fracamente positiva entre a produção das aflatoxinas B e G, estatísticamente significativa.
\end{abstract}

\section{INTRODUÇÃO}

A história da aflatoxina começou a ser contada quando STEVENS ef al. (1960) descreveram o aparecimento de uma nova doença em peruzinhos, nas granjas inglesas. As aves morriam geralmente dentro de uma semana sendo, seus sintomas, a perda de apetite, diminuição da mobilidade, fraqueza das asas, das pernas, etc. e provocando lesões necróticas no fígado. Não tendo sido possível identificar nenhum agente infeccioso, os autores suspeitaram que ela deveria ser de origem nutricional, pois que, mudando a alimentação muitas vezes cessava a mortalidade. Quase ao mesmo tempo esta doença foi descrita também por SWARBRICK (1960), WANNOP (1960), BLOUNT (1960) e WILEY (1960).

Veterinários e pesquisadores ingleses batizaram-na de doença $\alpha X_{\text {» }}$ dos perús e foi responsabilizada pela morte de mais de 100.000 peruzinhos entre maio e agosto de 1960 (BLOUNT, (1961).

* Entregue para publicação em 28/12/73.

* Professor de Disciplina, Departamento de Tecnologia Rural da ESALQ. 
Verificou-se, posteriormente, que uma partida de torta de amendoim, proveniente do Brasil, era o fator comum a todos os surtos de envenenamento (ASPLIN \& CARNAGHAN, 1961), ao mesmo tempo que, entre nós, AMARAL (1961) relacionava a morte de suinos com alimentos contendo torta de amendoim.

Isolado o princípio tóxico verificou-se que ele era composto de quatro substâncias às quais foram dadas o nome genérico de aflatoxina pois verificou-se que eram metabólitos tóxicos produzidos pelo fungo Aspergillus flavus Link (SARGEANT, et al., 1961) que se desenvolvia sobre o amendoim, após a colheita, sob condições favoráveis de umidade do amendoim e de temperatura e umidade relativa do ar.

As quatro substâncias foram denominadas de B1, B2, G1 e G2 pois produziam fluorescência azuis e verdes («blue» e «green») sob a luz ultra-violeta. Hoje são conhecidos outros metabólitos do grupo das aflatoxinas, ou sejam, as M1 e M2 (De IONGH et al., 1964 e ALLCROFT et al., 1966), as B2a e G2a (DUTTON \& HEATHCOTE, 1966) e as B3 e GM1 (DUTTON \& HEATHCOTE, 1969).

A aflatoxina é produzida pelo A. flavus, A. parasiticus e outros fungos, em quase todo o mundo, e em praticamente todos os substratos. RICHMOND ef al. (1962a e 1962b) demonstraram que os efeitos tóxicos se reproduziam em marrecos quando alimentados com soja, feijão ( runner bean»), semente de algodão e trigo, sobre os quais tinha crescido o A. flavus.

A importância do problema tem preocupado os governos de muitos países no sentido de conhecerem a ocorrência de aflatoxina em seus países, quer no amendoim, quer nas tortas e farelos. Já em 1961 na Nigéria, Mc DONALD \& HARKNESS (1963) fizeram uma investigação da presença da aflatoxina amendoim colhido em duas regiões distintas: Riverain e do Norte. Das 40 amostras analisadas muitas se apresentaram isentas da toxina, outras com valores até $0,5 \mathrm{ppm}$, apenas 3 ultrapassando este limite.

McDONALD \& A'BROOK (1963), no norte da Nigéria, pesquisaram a presença da aflatoxina em amendoim proveniente de vários experimentos de secagem nas safras de 1961 e 1962 e não encontraram valores acima de $0,5 \mathrm{ppm}$.

SELLSCHOP ef al. (1965) relataram um levantamento efetuado na Africa do Sul por uma equipe de técnicos que, em 1963/1964, examinou a ocorrência de aflatoxina em cerca de 2.000 amostras de amendoim e sub-produtos provenientes das províncias do Transvaal, do Natal e do Cabo, e do Estado Livre de Orange. As amostras de 1963 mostraram toxidez bastante elevada tendo, algumas partidas, apresentado $75 \%$ das amostras com mais de 2,0 ppm. Já em 1964 o número de amostras tóxicas, bem como o nível de toxidez decresceu, que, segundo aqueles autores, deve ter sido em virtude de medidas restritivas na aceitação de amendoim mofado.

PEERS (1965) apresentou uma das melhores pesquisas efetuadas neste campo ao relatar o trabalho por ele feito em Vom, no norte da Nigéria, durante os anos de 1963 a 1965, em dez partidas de amendoim provenientes da 
região de Zaria. Seu trabalho visou o controle da aflatoxina em tortas e farelos resultantes daquele amendoim e clestinados à composição do Arlac*, bem como verificar o efeito das medidas de melhoria das condições de secagem, armazenamento e seleção do amndoim. Estes estudos revelaram que a toxidez das primeiras partidas em 1963 foi em média de 0,34 ppm e que, com as melhorias introduzidas, a toxidez foi baixando até estabilizar-se, nas duas últimas partidas, em cerca de 0,02 ppm.

CROWTHER (1966), no período de fins de 1965 a meados de 1966, examinou 186 amostras de amendoim e farelos de amendoim provenientes de várias amostras em Gâmbia, cujos resultados variaram bastante. $\mathrm{Na}$ primeira partida $53 \%$ das amostras eram de toxidez «negativa», $19 \%$ «média» e $28 \%$ «alta», não se registrando nenhuma na categoria «muito alta». Todavia, em partidas de outra região, constatou farelos de toxidez muito elevada chegando até $40 \mathrm{ppm}$ de $\mathrm{B}_{1}$ e $30 \mathrm{ppm}$ de $\mathrm{G}_{1}$.

LIM \& YEAP (1966) levaram a efeito na Malásia em 1965 um pequeno levantamento em vários produtos como, amendoim descascado e tortas de amendoim, de gergelim, de côco, de soja e de milho importados e também do próprio país, num total de 69 amostras. Verificaram que apenas 25 estavam isentas, e das tóxicas, com predominância da aflatoxina $\mathrm{G}_{1}$ sobre a $\mathrm{B}_{1}$, muitas estavam ao nível de 2,0 ppm.

TOURY (1966) no Senegal, determinou aflatoxina em dez lotes de 246 amostras de amendoim e 9 de torta de amendeim, representando cerca de 200 toneladas de material. Apenas $16 \%$ das amostras de amendoim estavam tóxicas e destas apenas 2 tinham mais de $1,0 \mathrm{ppm}$. Quanto às tortas, todas estavam tóxicas porém, estavam na categoria «média», pois variavam de 0,05 a $0,12 \mathrm{ppm}$.

Entre nós MENEZES ef al. (1966), em trabalho que consideraram preliminar, determinaram aflatoxina em amendoim, tortas e farelos procedentes de várias fábricas do Estado de São Paulo. Mais de $90 \%$ da torta e farelo apresentaram teores elevados de aflatoxina tendo, os autores, considerado que apenas $3 \%$ delas estariam em condições de serem utilizadas na alimentação animal.

TANGO et al. (1967), trabalhando com amendoim das «águas» e da «seca» investigaram, entre outros, a incidência de aflatoxina e o grau de infestação de fungos. Os resultados mostraram uma maior inidência de aflatoxina na safra das «águas» com $59 \%$ das amostras nas categorias «Alta» e \&Muito Alta». Inversamente, encontraram um maior grau de infestação de fungos, na safra da «seca».

As propriedades carcinogênicas da aflatoxina foram constatadas em trutas (ASHLEY et al., 1964) ratos (WOGAN, 1965) e marrecos (SCHOENTAL, 1967). (*) Concentrado proteico destinado à infáncia no qual o farelo de amendoim entrava na pro-
porcăo de $33 \%$. 
Com relação ao homem, os primeiros indícios, de sua ação tóxica foram constatados por ZUCKERMAN \& FULTON (1966).

Sendo a aflatoxina um problema de grande importância, que pode atingir o homem tanto direta, pelo consumo de produtos contendo amendoim contaminado, como indiretamente através do leite produzido por animais que eventualmente se alimentem de farelos tóxicos, resolvemos investigar sua ocorrência em nosso Estado, através do farelo de amendoim, sub-produto da indústria de extração de óleo. Nesta investigação procurou-se esclarecer principalmente, os seguintes pontos: a) nível de toxidez dos farelos; b) variação da toxidez entre épocas de coleta das amostras e das fábricas, e c) possível correlação entre a produção das aflatoxinas $\mathrm{B}$ e $\mathrm{G}$.

Incluiu-se este último ítem por nada termos encontrado na literatura, com relação a ele, em trabalhos desta natureza.

\section{MATERIAL E MÉTODOS}

\section{Material}

O material utilizado para execução deste trabalho constou de 116 amostras de farelo de amendoim da safra de 1966/67 coletadas em 17 fábricas de óleo, localizadas na região servida pela Companhia Paulista de Estradas de Ferro, compreendida entre Bauru e Adamantina, no Estado de São Paulo.

As amostras foram coletadas em quatro épocas a saber: março e maio, representando material proveniente da safra das «águas» num total de 68 amostras e julho e setembro, representando material da safra da «seca», num total de 48 amostras visto que, nesta safra, apenas 12 fábricas trabalharam.

De cada fábrica foi retirada uma amostra de cerca de $1 \mathrm{~kg}$ representativa de todo o material existente na fábrica e recolhida em sacos plásticos.

As fábricas foram identificadas por números e as épocas de coleta com os nomes dos meses em que a amostra foi retirada. letas.

As análises foram efetuadas nos dias imediatos após o término das co-

\section{Métodos}

\section{Preparo das amostras}

As amostras foram trituradas passando-se quatro a cinco vezes em moinho de discos ( $\ll$ Disc mill», modelo 4E, The Straub Co., Philadelphia, EEUU.) para obtenção da finura adequada para a análise sendo, posteriormente, passadas em peneiras de crivo de 841 micra $(20 \ll m e s h »)$. Em seguida as amostras foram divididas em duas sub-amostras: $\mathbf{a}$ e $\mathbf{b}$, que foram novamente guardadas em sacos plásticos, até o momento de serem analisadas.

\section{Extração da toxina}

De cada sub-amostra, daqui para frente denominadas simplesmente de amostra, foram tomados $20 \mathrm{~g}$, dos quais foi extraida a toxina com clorofór- 
mio, de acordo com o método de LEE (1965). O filtrado obtido foi denominado de solução $X$. Desta foram tomadas $8 \mathrm{ml}$ e diluidos a $100 \mathrm{ml}$ com clorofórmio: solução $\mathbf{Y}$.

\section{Preparo das cromatoplacas}

Placas de vidro de $10 \times 20 \mathrm{~cm}$, com camada de 500 micra de silicagel-G, foram preparadas segundo a técnica de COOMES \& FEUELL (1965).

\section{Cromatografia do extrato}

A $2 \mathrm{~cm}$ da base das cromatopiacas foram colocadas as seguintes alíquotas: $20 \mu 1$ da solução $X$ e $25,10,5$ e $2,5 \mu l$ da solução $Y$, além de uma alíquota de concentração conhecida, para comparação, e desenvolvidas com o solvente clorofórmio-metanol (95:5), até $10 \mathrm{~cm}$ acima do local das amostras, em câmaras saturadas.

\section{Determinação do teor de aflatoxina e sua correspondente toxidez}

As placas, após a cromatografia, foram examinadas em câmara escura, a distância de $30 \mathrm{~cm}$ de uma lámpada ultra-violeta Philips tipo HPW, 125 watt, com emissão máxima em $365 \mathrm{r}$ m e observada a presença ou ausência de manchas fluorescentes azul-violeta, das aflatoxinas $\mathrm{B} 1+\mathrm{B} 2$ num $\mathrm{Rf}=0,50-0,55$ e esverdeada, das aflatoxinas $\mathrm{G} 1+\mathrm{G} 2$ num $\mathrm{Rf}=0,45-0,50$.

O cálculo da concentração foi efetuado segundo COOMES \& FEUELL (1965). Este sistema forneceu resultados entre dois limites de concentração. Assim, a menor alíquota em que foi observada fluorescência, nos forneceu o limite inferior. Para o limite superior foi tomada a alíquota seguinte em que não foi observada fluorescência. Nos casos em que foi observada fluorescência na menor das alíquotas, a solução $Y$ foi rediluida e recromatografada para verificarmos o limite superior. Estes limites, em função da menor alíquota em que foi observada fluorescência, foram:

\begin{tabular}{rrcrr}
\multicolumn{2}{c}{ Alíquota } & B1 + B2 & G1 & + G2 \\
20 & $\mu$ l sol. $X$ & $0,10-1,00$ & 0,075 & $-0,75$ \\
25 & $\mu$ l sol. $Y$ & $1,00-2,50$ & 0,75 & $-1,87$ \\
10 & $\mu 1$ sol. $Y$ & $2,50-5,00$ & 1,87 & $-3,75$ \\
5 & $\mu$ l sol. $Y$ & $5,00-10,00$ & 3,75 & $-7,50$ \\
2,5 & $\mu l$ sol. $Y$ & $10,00-20,00$ & 7,50 & $-15,00$
\end{tabular}

Para avaliação da toxidez das amostras os resultados foram enquadrados nas categorias de toxidez estabelecidas pelo TROPICAL PRODUCTS INSTTTUTE (1962) e que consta do QUADRO 1. 
Quadro 1 - Relação entre a concentração de aflatoxina $B_{1}$ e toxidez do material.

\begin{tabular}{ll}
\hline Categoria de toxidez & Nivel de aflatoxina $B_{1}$ \\
\hline Muito Alta & Acima de $1,00 \mathrm{ppm}$ \\
Alta & Entre 0,25 e $1,00 \mathrm{ppm}$ \\
Média & Entre 0,05 e $0,25 \mathrm{ppm}$ \\
Baixa ou Negativa & Abaixo de $0,05 \mathrm{ppm}$ \\
\hline
\end{tabular}

A nossa escala de categorias foi ligeiramente modificada para maior facilidade dos trabalhos de análise. Além disso, quando do início dos nossos trabalhos, verificamos que os valores encontrados eram bastante elevados e por isso resolvemos esmiuçar mais a categoria «Muito Alta» ou seja, farelos com mais de $1,00 \mathrm{ppm}$. Por esse motivo subdividimo-la em quatro níveis.

\section{Análise estatística}

Para a computação das médias foi usado o centro dos intervalos dos níveis (PEERS, 1965) com as variáveis transformadas em $\log (x+1)$ de acordo com SNEDECOR (1956) e STEEL \& TORRIE (1960).

$O$ cálculo da correlação entre as aflatoxinas $B$ e $G$, foi feito conforme SNEDECOR (1956) também com as variáveis transformadas em $\log (x+1)$.

Para a análise da variância dos dados foi considerado o modelo:

$$
\mathrm{Y}=\mathrm{u}+\mathrm{fi}+\mathrm{sj}+(\mathrm{fs})_{\mathrm{ij}}+\mathrm{m}_{\mathrm{k}(\mathrm{j})}+(\mathrm{fm})_{\mathrm{ik}(\mathrm{j})}+\mathrm{a}_{\mathrm{l}(\mathrm{ijk})}
$$

onde: $\quad \mathrm{i}=1,2,3, \ldots \ldots \ldots \ldots \ldots . . .17$.

$\mathrm{j}=1,2$.

$\mathrm{k}=1,2$.

$1=1,2$.

tendo os efeitos, todos aleatórios, o seguinte significado:

$\mathrm{f}=$ efeito de fábricas

$\mathbf{s}=$ efeito de safras

$(\mathrm{fs})=$ efeito da interação fábrica $\mathrm{x}$ safra

$\mathrm{m}=$ efeito do mês (ou época) dentro da safra

$(\mathrm{fm})=$ efeito da interação fábrica $\mathbf{x}$ mês dentro da safra

$\mathrm{a}=$ ef eito da amostra.

A análise da variância foi feita para estimar as variâncias relativas a cada ef eito. Os dados também foram transformados em $\log (x+1)$. 
A esperança matemática dos quadrados médios foi computada conforme BENNETT \& FRANKLIN (1954).

O centro dos intervalos dos níveis, com os quais foram feitos os cálculos acima referidos foram os seguintes:

\begin{tabular}{|c|c|c|c|c|c|}
\hline \multicolumn{3}{|c|}{ Aflatoxina B } & \multicolumn{3}{|c|}{ Aflatoxina $G$} \\
\hline \multicolumn{2}{|r|}{ Intervalo } & \multirow{2}{*}{$\begin{array}{l}\text { Centro } \\
0,025\end{array}$} & \multicolumn{2}{|c|}{ Intervalo } & \multirow{2}{*}{$\begin{array}{c}\text { Centro } \\
0,0375\end{array}$} \\
\hline 0,00 & $-\quad 0,05$ & & $0.00-$ & 0,075 & \\
\hline 0.05 & 0,10 & 0,075 & $0,075-$ & 0,75 & 0,4125 \\
\hline 0,10 & 1,00 & 0,55 & $0,75-$ & 1,87 & 1,31 \\
\hline 1,00 & 2.50 & 1.75 & $1.87-$ & 3,75 & 2,81 \\
\hline 2.50 & 5,00 & 3,75 & $3,75-$ & 7,50 & 5,625 \\
\hline 5,00 & 10,00 & 7.50 & $7.50-$ & 15,00 & 11,25 \\
\hline 10,00 & $-\quad 20.00$ & 15,00 & - & - & - \\
\hline
\end{tabular}

\section{RESULTADOS E DISCUSSAO}

\section{Resultados}

Os resultados das análises são os constantes das quadros de números 2 a 15.

\section{Discussão}

Neste trabalho os resultados são apresentados em níveis de toxidez, pois pela sua natureza, um levantamento, desejou-se saber apenas a extensão e o nível de incidência da toxina. Esta forma de apresentação é comum entre os autores como pode ser observado em PEERS (1965), SELLSCHOP et al. (1965), LIM \& YEAP (1966), TOURY (1966), MENEZES ef al. (1966) e TANGO et al. (1967).

Os níveis de toxidez estão intimamente relacionados com os testes biológicos em marrequinhos de um dia pois, é evidente que os números absolutos do conteúdo de aflatoxina não darão qualquer informação de sua toxidez a não ser quando relacionados com ses efeitos nos referidos testes.

Conforme pode-se observar nos quadros de números 2 a 5 , a aflatoxina esteve presente em todas as amostras e variou entre os limites de 0,1 e 20,0 ppm para as aflatoxinas B e entre 0,075 e 7,5 ppm para as G.

Baseado nos resultados apresentados para as aflatoxinas $B$ podemos verificar, pelo QUADRO 11, que não temos nenhuma incidência nas categorias «Baixa ou Negativa» e «Média», que $10,35 \%$ estão na categoria «Alta» e que $89,65 \%$ estão na «Muito Alta». 
A toxidez dos farelos foi como se vè, bastante elevada. Para que se tenha uma idéia do significado dos resultados encontrados pode-se citar que a concentração de aflatoxina $B_{1}$ no farelo que ocasionou a mortandade de peruzinhos na Inglaterra em 1960 , estava entre 2,0 e 5,0 ppm. Como pode-se observar, ainda com dados do QUADRO 11, 65,51\% das amostras estavam com mais de 2,5 ppm. Estes resultados estão de acordo com os encontrados entre nós por MENEZES et al. (1966) e por TANGO et al. (1967).

Tomando por base a recomendação de instituições que pesquisaram extensivamente o assunto (ANONIMO, 1969), pela qual os farelos ou tortas com mais de $1,00 \mathrm{ppm}$ praticamente não podem ser utilizados na formulação de rações animais em nenhuma proporção, não poderíamos aproveitar mais do que $10,35 \%$ do material examinado.

A situação em outros países é bastante variável, conforme pode-se observar nos trabalhos de McDONALD \& HARKNESS (1963) e Mc DONALD \& A'BROOK (1963), ambos na Nigéria, SELLSCHOP et al. (1965) na África do Sul, PEERS (1965) em Vom no norte da Nigéria, TOURY (1966) no Senegal, além de outros. Todavia pode-se constatar facilmente que o nível de toxidez dos farelos por nós examinados foi bem mais elevado que o encontrado naqueles países, com algumas exceções.

Pela análise da variância dos resultados pode-se verificar que não houve diferença de comportamento entre as fábricas, tanto para as aflatoxinas B como para as G. Houve influência da safra, significativa ao nível de $1 \%$ de probabilidade apenas com relação às aflatoxinas $\mathrm{B}$. A distribuição das incidências por safras e por níveis para os dois grupos de aflatoxinas pode ser vista nos quadros de números 13 e 14 os quais mostram que essa distribuição realmente variou.

Pode-se notar claramente pelas médias calculadas (QUADRO 15) que houve uma grande diferença entre as «águas» e a «seca».

A época ou mês de coleta dentro de uma mesma safra também não variou significativamente. A interação mês vs. fábrica dentro de uma mesma safra variou significativamente ao nível de $1 \%$ para as aflatoxinas $\mathrm{B}$ e ao nível de 5\% para as $\mathrm{G}$. Isto significa um comportamento bastante irregular das amostras entre as fábricas de uma época de coleta para outra dentro da mesma safra. A interação fábrica vs. safra não variou significativamente com relação às aflatoxinas $B$ mas sim com relação às $G$, ao nível de $5 \%$.

Pelos quadros de números 8 e 9 pode-se verificar que, com relação às aflatoxinas B, o maior fator de variação foi realmente a safra, com 62,94\% do total, ao passo que com as $\mathrm{G}$ os fatores de variação estiveram mais distribuidos.

A maior variação devida à safra já era mais ou menos esperada poìs, nas «águas» as condições são muito mais favoráveis ao desenvolvimento de fungos, e em particular do A. flavus, do que na «seca». Embora o valor médio das «águas» tenha sido bem mais elevado, e o da «seca» também esteve num nível alto. 
Todos os farelos examinados eram tóxicos e apresentaram ambos os grupos de aflatoxinas. Os valores encontrados para as $B$ foram sempre mais elevados que os das $G$ na mesma amostra e porisso ocorreu-nos investigar também se havia qualquer correlação entre a produção daqueles me: tabólitos visto que, em trabalhos de campo, não há referência na literatura sobre o assunto. O resultado obtido no estudo dessa possível correlação (QUADRO 10) mostrou ter havido uma correlação fracamente positiva, estatisticamente significativa.

\section{CONCLUSOES}

Dos resultados obtidos no presente trabalho pôde-se tirar as seguintes conclusões:

1) A incidência da aflatoxina foi geral na região Paulista Nova pois todas as amostras estavam tóxicas.

2) O nível de toxidez foi, no geral, muito elevado, com algumas amostras chegando a $20,0 \mathrm{ppm}$.

3) Os farelos da safra das «águas» apresentaram toxidez bem maior (média de 5,50 ppm) que os da «seca» (média de 1,76 ppm).

4) Apenas $10,35 \%$ do material analisado poderia ser utilizado na alimentação animal pois o restante enquadrou-se na categoria «Muito Alta» com mais de $1,0 \mathrm{ppm}$.

5) Houve uma correlação fracamente positiva entre as aflatoxinas B eG.

\section{SUMMARY}

\section{OCCURRENCE OF AFLATOXIN IN PEANUT FLOUR IN THE REGION} PAULISTA NOVA, IN THE STATE OF SAO PAULO.

In the present work, the occurence of aflatoxins $B$ and $G$ as well as a possible correlation between both were studied in 116 samples of peanut flour from 17 oil mills of the region Paulista Nova, in the State of São Paulo.

The samples were obtained in four collections representing material from two crops in two different seasons: March and May in the rainy season and July and September in the dry season.

From the results it was concluded that: a) the occurence of aflatoxin was generalized in that region, for all the samples were toxic; b) the toxicity level was found to be very high (figures from 0.1 to $20.0 \mathrm{ppm}$ ) being much higher in the rainy season-average of $5.50 \mathrm{ppm}$ - against $1.76 \mathrm{ppm}$ in the dry season; c) it was considered that only $10.35 \%$ of the material examined could be utilized for admixturing in feedstuffs; d) it was found a weakly positive correlation in the production of the aflatoxins $B$ and $G$. 


\section{AGRADECIMENTOS}

Agradecemos à FAPESP pelo auxílio financeiro concedido para a realização desta pesquisa e à UNICEF pelo auxílio em drogas e aparelhos. Agradecemos também ao prof. Roland Vencovsky e ao acadêmico de agronomia Isaias 0. Geraldi pela execução das análises estatísticas.

\section{LITERATURA CITADA}

ALLCROFT, R., H. ROGERS, G. LEWIS, J. NABNEY \& P. E. BEST, $1966-$ Metabolism of aflatoxin in sheep: excretion of the milk toxin. Nature, 209 (5019): 154-155.

AMARAL, L. B. S., 1961 - Torta de amendoim e morte de suinos. O Biológico, $27(3): 63$.

ANONIMO, 1969 - Informationsdienst Futter und Fütterung. Ed. Fachverband der Futtermittelindustrie e. V., Hamburg.

ASHLEY, L. M., J. E. HALVER \& G. N. WOGAN, 1964 - Hepatoma and aflatoxicosis in trout. Fed Proc. 23: 105.

ASPLIN, F. D. \& R. B. A. CARNAGHAN 1961 - The toxicity of certain groundnut meals for poultry with special reference to their effect on ducklings and chickens. Vet. Rec., 73: 1215-1219.

BENNETT, C. A. \& N. L. FRANKLIN, 1963 - Statistical Analysis in Chemistry and the Chemical Industry. John Wiley \& Sons, Inc., N. York, 724 pp.

BLOUNT, W. P., 1960 - Disease of turkey poults. Vet. Rec., 72 (38): 786.

BLOUNT, W. P., 1961 -- Turkey "X" disease. Turkeys, 9(2): 52, 55-58, 61, 67.

COOMES, T. J. \& A. J. FEUELL, 1965 - Recommended procedures for the detection of aflatoxin $B_{1}$ in groundnuts and groundnut materials. Trop. Prod. Inst. Report. n. ${ }^{9}$ G13. London, 24 pp.

CROWTHER, P. C., 1966 - Report of the Produce Chemist, $4^{\text {th }}$ november 1965 - $4^{\text {th }}$ may 1966. The Laboratory, Dept. of Agriculture, Yundum Experimental Station, The GAMBIA. $15 \mathrm{pp}$.

De IONGH, H., R. O. VLES \& J. G. Van PELT, 1964 - Milk of mammals fed an aflatoxin - containing diet. Nature, 202: 466-467.

DUTTON, M. F. \& J. G. HEATHCOTE, 1966 - Two new aflatoxins. Biochem. J. 101: 21P-22P.

DUTTON, M. F. \& J. G. HEATHCOTE, 1969 - Some interesting relationship between the new aflatoxins and their associated metabolites. The J. South African Chem. Inst., XXII: 5107-5118.

LEE, W. V., 1965 - Quantitative determination of aflatoxin in groundnut products. Analyst (Lond.) 90 (1070) : 305-307.

LIM, H. K. \& G. S. YEAP, 1966 - The occurrence of aflatoxin in Malayan imported oil cakes and groundnut kernels. The Malaysian Agric. J., 45 (3): 232-244.

MCDONALD, D. \& J. A'BROOK, 1963 - Growth of Aspergillus flavus and production of aflatoxin in groundnuts. Part. III. Trop. Sci., 5 (4) : 208-214.

MCDONALD, D. \& C. HARKNESS, 1963 - Growth of Aspergillus flaves and production of aflatoxin in groundnuts. Part. II. Trop. Sci., 53 (3) : 143-154. 
MENESES, T. J. B., J. S. TANGO, F. A. S. COELHO \& C. G. TEIXEIRA, 1966 - Ocorrência do Aspergillus flavus e da aflatoxina em sementes e farelo de amendoim. Apresentado à XVIII Reunião Anual da S. B. P. C., Blumenau, S.C. 1966.

PEERS, F. C., 1965 - Summary of the work done at-Vom (Northern Nigeria) on aflatoxin levels in grondnut flour and Arlac. Nutr. Docum. Aflatoxin/8. WHO/FAO/UNICEP - P. A. G. 1965 Meeting, Rome.

RICHMOND, J. W., N. H. SUTCLIFFE, N. W. R. DANIELS, P. W. RUSSELEGGITT \& J. B. M. COPPOCK, 1962a - Factors other groundnut relating to "turkey X disease". Vet. Rec., it (18): 544-545.

RICHMOND, J. W., N. H. SUTCLIFFE, N. W. R. DANIELS, P. W. RUSSELEGGITT \& J. B. M. COPPOCK, 1962b - Factors other than groundnut in the production of aflatoxin. Vet. Rec., it/ (35) 962.

SARGEANT, K., A. SHERIDAN, J. O'KELLY \& R. B. A. CARNAGHAN, 1961 - Toxicity associated with certain samples of groundnuts. Nature, 192: 1096* 1097.

SCHOENTAL, R., 1967 - Aflatoxins. In: Annual Rev. Pharmac. 7: 343-356.

SELLSCHOP, J. P. F., N. P. J. KRIEK \& J. C. G. du PREEZ, 1965 - Distribution and degree of occurrence of aflatoxin in groundnuts and groundnut products. Symp. Mycotoxins Foodstuffs, Agric. Aspects, Febr. 1965, Pretoria, South Africa: 9-17.

SNEDECOR, G. W., 1956 - Statistical Methods. The Iowa State College Press, Ames, Iowa, EUA., $5^{*} \mathrm{Ed} ., 534 \mathrm{pp}$.

STEEL, R. G. D. \& J. H. TORRIE, 1960 - Principles and Procedures of Statistics. McGraw Hill Book Co., Inc., N. York, 481 pp.

STEVENS, A. J., C. N. SAUNDERS, J. B. SPENCE \& A. G. NEWHAM, 1960 - Investigations into "diseases" of turkey poults. Vet. Rec. $72:$ (31) 627-628.

SWARBRICK, O., 1960 - "Disease" of turkey poults. Vet. Rec. $\% 2$ (33) : 671.

'TANGO, J. S., T. J. B. MENEZES \& C. G. TEIXEIRA, 1967 - Levantamento da ocorrência da aflatoxina em sementes de amendoim nas safras das águas e da seca de 1965. Apresentado à XIX Reunião Anual da S. B. P. C., Rio de Janeiro, 1967.

TOURY, J., 1966 - Rapport sur l'Operation Exagraf. In "Rapport sur les recher ches effectuées sur l'aflatoxine au cours de l'année 1965 - 1966. O.R.A.N.A., Dakar, Senegal, $12 \mathrm{pp}$.

TROPICAL PRODUCTS INSTITUTE, 1962 - Aflatoxin in groundnuts and groundnut products. Interpretation of physico-chemical and biological test results. T.P.I., Ministry of Overseas Development, Londres, $1 \mathrm{p}$.

WANNOP, C. C., 1960 - Disease of turkey poults. Vet. Rec., i2 (33) : 671-672.

WILEY, J. R., 1960 - "Disease" of turkey poults. Vet. Rec., 72 (38) : 786-787.

WOGAN, G. N., 1965 - Experimental toxicity and carcinogenicity of aflatoxins. In "Mycotoxins in Foodstuffs". Ed. G. N. Wogan, The M. I. T. Press, Cambridge, Mass. EE.UU., p. 163-173.

ZUCKERMAN, A. J. \& F. FULTON, 1966 - Acute toxic effects of aflatoxin on human embryo liver cells in culture. Brit. Med. J: 2 90-91. 
Quadro 2 - Ocorrência das aflatoxinas B e G em amostras da safra das “águas" MARÇO (expresso em ppm)

\begin{tabular}{|c|c|c|c|c|c|c|}
\hline \multirow{3}{*}{$\begin{array}{r}\begin{array}{r}\text { Fá- } \\
\text { bri- } \\
\text { ca }\end{array} \\
\text { no } \\
\quad 1\end{array}$} & \multicolumn{3}{|c|}{ Amostra a } & \multicolumn{3}{|c|}{ Amostra $b$} \\
\hline & \multicolumn{2}{|r|}{ B } & $\mathrm{G}$ & \multicolumn{2}{|c|}{ B } & G \\
\hline & 5,0 & $-10,0$ & $0,75-1,87$ & 5,0 & $-10,0$ & $0,75-1,87$ \\
\hline 2 & 2,5 & $-\quad 5,0$ & $0,075-0,75$ & 2,5 & $-\quad 5,0$ & $0,075-0,75$ \\
\hline 3 & 10,0 & $-20,0$ & $1,87-3,75$ & 5,0 & $-10,0$ & $1,87-3,75$ \\
\hline 4 & 2,5 & $-\quad 5,0$ & $0,075-0,75$ & 2,5 & $-\quad 5,0$ & $0,075-0,75$ \\
\hline 5 & 2,5 & $-5,0$ & $0,075-0,75$ & 2,5 & $-\quad 5,0$ & $0,075-0,75$ \\
\hline 6 & 5,0 & $-10,0$ & $1,87-3,75$ & 5,0 & $-10,0$ & $1,87-3,75$ \\
\hline 7 & 2,5 & $-\quad 5,0$ & $0,075-0,75$ & 2,5 & $-\quad 5,0$ & $0,075-0,75$ \\
\hline 8 & 1,0 & $-2,5$ & $0,075-0,75$ & 1,0 & $-2,5$ & $0,075-0,75$ \\
\hline 9 & 2,5 & $-\quad 5,0$ & $0,075-0,75$ & 2,5 & $-5,0$ & $0,075-0,75$ \\
\hline 10 & 5,0 & $-10,0$ & $0,075-0,75$ & 2,5 & $-\quad 5,0$ & $0,075-0,75$ \\
\hline 11 & 5,0 & $-10,0$ & $0,075-1,87$ & 5,0 & $-10,0$ & $0,075-0,75$ \\
\hline 12 & 2,5 & $-5,0$ & $0,75-0,75$ & 5,0 & $-10,0$ & $0,75-1,87$ \\
\hline 13 & 5,0 & $-10,0$ & $0,075-0,75$ & 5,0 & $-10,0$ & $0,075-0,75$ \\
\hline 14 & 5,0 & $-10,0$ & $0,075-0,75$ & 5,0 & $-10,0$ & $0,075-0,75$ \\
\hline 15 & 2,5 & $-5,0$ & $0,075-0,75$ & 5,0 & $-10,0$ & $0,075-0,75$ \\
\hline 16 & 5,0 & $-10,0$ & $1,87-3,75$ & 5,0 & $-10,0$ & $1,87-3,75$ \\
\hline 17 & 2,5 & $-\quad 5,0$ & $0,075-0,75$ & 2,5 & $-\quad 5,0$ & $0,075-0,75$ \\
\hline
\end{tabular}


Quadro 3 - Ocorrência das aflatoxinas B e G em amostras da safra das "águas" MAIO (expresso em ppm)

\begin{tabular}{|c|c|c|c|c|c|c|}
\hline \multirow{3}{*}{$\begin{array}{l}\text { Fábri- } \\
\text { ca } \\
\text { no } \\
1\end{array}$} & \multicolumn{3}{|c|}{ Amostra a } & \multicolumn{3}{|c|}{ Amostra $b$} \\
\hline & \multicolumn{2}{|r|}{$B$} & $G$ & \multicolumn{2}{|r|}{ B } & $G$ \\
\hline & 10,0 & $-20,0$ & $3,75-7.50$ & 10,0 & $-20,0$ & $1,87-3,75$ \\
\hline 2 & 1,0 & $-\quad 2,5$ & $0,075-0,75$ & 2,5 & $-\quad 5,0$ & $0,075-0,75$ \\
\hline 3 & 5,0 & $-10,0$ & $1,87-3,75$ & 5,0 & $-10,0$ & $1,87-3,75$ \\
\hline 4 & 2,5 & $-5,0$ & $0,075-0,75$ & 5,0 & $-10,0$ & $0,075-0,75$ \\
\hline 5 & 10,0 & $-20,0$ & $1,87-3,75$ & 5,0 & $-10,0$ & $1,87-3,75$ \\
\hline 6 & 5,0 & $-10,0$ & $1,87-3,75$ & 5,0 & $-10,0$ & $1,87-3,75$ \\
\hline 7 & 5,0 & $-10,0$ & $0,75-1,87$ & 5,0 . & $-10,0$ & $0,75-1,87$ \\
\hline 8 & 5,0 & $-10,0$ & $0.75-1.87$ & 5.0 & $-10,0$ & $1,87-3.75$ \\
\hline 9 & 5,0 & $-10,0$ & $0.075-0,75$ & 5,0 & $-10,0$ & $0,075-0,75$ \\
\hline 10 & 2,5 & $-\quad 5,0$ & $0,075-0,75$ & 2,5 & $-\quad 5,0$ & $0,075-0,75$ \\
\hline 11 & 10,0 & $-20,0$ & $0.075-0.75$ & 5.0 & $-10,0$ & $0,075-0,75$ \\
\hline 12 & 2,5 & $-\quad 5,0$ & $0,75-1,87$ & 2,5 & $-\quad 5,0$ & $0,75-1,87$ \\
\hline 13 & 1,0 & $-\quad 2.5$ & $0,075-1,87$ & 2.5 & $-\quad 5.0$ & $0.075-0.75$ \\
\hline 14 & 2,5 & $-\quad 5.0$ & $0,075-0,75$ & 2.5 & 5,0 & $0,075-0,75$ \\
\hline 15 & 2.5 & $-\quad 5.0$ & $0,075-0.75$ & 2.5 & $-\quad 5.0$ & $0,00-0,075$ \\
\hline 16 & 5,0 & $-10,0$ & $1,87-3.75$ & 2.5 & $-\quad 5.0$ & $0,075-0,75$ \\
\hline 17 & 2,5 & $-\quad 5,0$ & $0.075-0.75$ & 2.5 & $-\quad 5,0$ & $0.075-0.75$ \\
\hline
\end{tabular}


Quadro 4 - Ocorrencia das aflatoxinas B e G em amostras da safra da "seca" JULHO (expresso em ppm)

\begin{tabular}{rcccccc}
\hline \begin{tabular}{l} 
Fábri- $\begin{array}{c}\text { Amostra } \\
\text { no }\end{array}$ \\
\cline { 2 - 6 }
\end{tabular} & \multicolumn{3}{c}{ B } & G & \multicolumn{3}{c}{ Amostra b } \\
\hline 2 & 0,1 & $-1,0$ & $0,075-0,75$ & $1,0-2,5$ & $0,075-0,75$ \\
3 & 1,0 & $-2,5$ & $0,75-1,87$ & $1,0-2,5$ & $0,75-1,87$ \\
8 & 1,0 & $-2,5$ & $0,75-1,87$ & $2,5-5,0$ & $1,87-3,75$ \\
9 & 2,5 & $-5,0$ & $0,75-1,87$ & $2,5-5,0$ & $0,075-0,75$ \\
10 & 0,1 & $-1,0$ & $0,075-0,75$ & $1,0-2,5$ & $0,075-0,75$ \\
11 & 2,5 & $-5,0$ & $0,75-1,87$ & $2,5-5,0$ & $0,075-0,75$ \\
12 & 0,1 & $-1,0$ & $0,075-0,75$ & $0,1-1,0$ & $0,075-0,75$ \\
13 & 2,5 & $-5,0$ & $0,075-0,75$ & $5,0-10,0$ & $0,075-0,75$ \\
14 & 1,0 & $-2,5$ & $0,075-0,75$ & $0,1-1,0$ & $0,075-0,75$ \\
15 & 1,0 & $-2,5$ & $0,075-0,75$ & $1,0-2,5$ & $0,075-0,75$ \\
16 & 1,0 & $-2,5$ & $0,075-0,75$ & $1,0-2,5$ & $0,075-0,75$ \\
17 & 1,0 & $-2,5$ & $0,075-0,75$ & $1,0-2,5$ & $0,075-0,75$ \\
\hline
\end{tabular}

Obs. - As fábricas de nọs. 1, 4, 5, 6 e 7 não trabalharam com a safra da "seca". 
Quadro 5 - Ocorrência das aflatoxinas B e G em amostras da safra da "seca" SETEMBRO (expresso em ppm)

\begin{tabular}{|c|c|c|c|c|c|c|}
\hline \multirow{2}{*}{$\begin{array}{l}\text { Fábri- } \\
\text { ca } \\
\text { n! }\end{array}$} & \multicolumn{3}{|c|}{ Amostra a } & \multicolumn{3}{|c|}{ Amostra $b$} \\
\hline & & B & $\mathrm{G}$ & & B & G \\
\hline 2 & 0,1 & $-1,0$ & $0,075-0,75$ & 0,1 & $-1,0$ & $0,075-0,75$ \\
\hline 3 & 0,1 & $-1,0$ & $0,075-\quad 0,75$ & 1,0 & $-2,5$ & $0,075-0,75$ \\
\hline 8 & 2,5 & $-5,0$ & $0,075-0,75$ & 1,0 & $-2,5$ & $0,075-0,75$ \\
\hline 9 & 2,5 & $-5,0$ & $0,075-0,75$ & 1,0 & $-2,5$ & $0,075-0,75$ \\
\hline 10 & 1,0 & $-2,5$ & $0,075-0,75$ & 2,5 & $-5,0$ & $0,075-0,75$ \\
\hline 11 & 1,0 & $-2,5$ & $0,075-0,75$ & 1,0 & $-2,5$ & $0,075-0,75$ \\
\hline 12 & 1,0 & $-2,5$ & $0,75-1,87$ & 2,5 & $-5,0$ & $0,075-0,75$ \\
\hline 13 & 1,0 & $-2,5$ & $0,075-0,75$ & 1,0 & $-2,5$ & $0,075-0,75$ \\
\hline 14 & 0,1 & $-1,0$ & $0,075-0,75$ & 0,1 & $-1,0$ & $0,075-0,75$ \\
\hline 15 & 0,1 & $-1,0$ & $0,075-0,75$ & 0,1 & $-1,0$ & $0,075-0,75$ \\
\hline 16 & 1,0 & $-2,5$ & $0,075-0,75$ & 1,0 & $-2,5$ & $0,075-0,75$ \\
\hline 17 & 2,5 & $-5,0$ & $0,075-0,75$ & 1,0 & $-2,5$ & $0,075-0,75$ \\
\hline
\end{tabular}

Obs. - As fábricas de nos. 1, 4, 5, 6 e 7 não trabalharam com a safra da "seca". 
Quadro 6 - Análise da variância das aflatoxinas B

\begin{tabular}{lrrcc}
\hline Causa de variação & G.L. & S.Q. & Q.M. & F \\
\hline Fábricas (F) & 11 & 0,795257 & 0,072296 & 1,35 n.s. \\
Safras (S) & 1 & 2,815658 & 2,815658 & $35,96^{* *}$ \\
Interação F x S & 11 & 0,587214 & 0,053383 & 1,08 n.s. \\
Mes dentro de safra (M) & 2 & 0,052574 & 0,026287 & 0,53 n.s. \\
M x F dentro de S & 22 & 1,088657 & 0,049484 & $4,06^{* *}$ \\
Amostragem & 48 & 0,584540 & 0,012178 & \\
\hline T O T A L & 95 & 5,923900 & - & - \\
\hline ** Significativo a nif
\end{tabular}

** Significativo ao nível de $1 \%$ de probabilidade.

Quadro 7 - Análise da variância das aflatoxinas G

\begin{tabular}{lrlll}
\hline Causa de variação & \multicolumn{5}{l}{} \\
\hline Fábricas (F) & 11 & 0,745436 & 0,067767 & 1,84 n.s. \\
Safras (S) & 1 & 0,086983 & 0,086983 & 1,84 n.s. \\
Interação F x S & 11 & 0,404412 & 0,036765 & $2,57^{*}$ \\
Mes dentro safra (M) & 2 & 0,036464 & 0,018232 & 1,27 n.s. \\
M x F dentro de S & 22 & 0,315163 & 0,014326 & $2,85^{*}$ \\
Amostragem & 48 & 0,241096 & 0,005023 & \\
\hline T O T A L & 95 & 1,829554 & - & - \\
\hline
\end{tabular}

* Significativo ao nível de $5 \%$ de probabilidade. 
Quadro 8 - Estimativa da variância de cada fator de variação para as aflatoxinas B

\begin{tabular}{l|l|r|r}
\hline Fator de variação & \multicolumn{2}{|c|}{$\sigma^{2}$} & $\%$ \\
\hline Fábricas (F) & $\sigma_{\mathrm{f}}^{2}$ & 0,002364 & 2,56 \\
Safras (S) & $\sigma^{2} \mathrm{~s}$ & 0,058030 & 62,94 \\
F x S & $\sigma^{2} \mathrm{fs}$ & 0,000974 & 1,06 \\
Meses d. safra (M) & $\sigma^{2} \mathrm{~m}$ & $-0,000966$ & 0,00 \\
M x F dentro de S & $\sigma^{2} \mathrm{mf}$ & 0,018653 & 20,23 \\
Amostragem & $\sigma^{2}$ & 0,012178 & 13,21 \\
\hline T O T A L & & 0,092199 & 100,0 \\
\hline
\end{tabular}

Quadro 9 - Estimativa da variância de cada fator de variação para as aflatoxinas G.

\begin{tabular}{l|l|l|r}
\hline Fator de variação & \multicolumn{2}{|c|}{$\hat{G}^{2}$} & $\%$ \\
\hline Fábrica (F) & $G^{2}$ & 0,003875 & 19,10 \\
Safras (S) & $G^{2}$ & 0,000964 & 4,75 \\
F x S & $G^{2} \mathrm{f} s$ & 0,005609 & 27,65 \\
Meses d. safra (M) & $G^{2} \mathrm{~m}$ & 0,000162 & 0,80 \\
M x F dentro de S & $G^{2} \mathrm{~m} \mathrm{f}$ & 0,004651 & 22,93 \\
Amostragem & $G^{2}$ & 0,005023 & 24,76 \\
\hline T O T A L & - & 0,020284 & 100,00 \\
\hline
\end{tabular}

Quadro 10 - Correlação entre as aflatoxinas B e G

$$
r=0,37^{*}
$$


Quadro 11 - Distribuição do número de incidências das aflatoxinas $\mathrm{B}$, por níveis $\mathrm{e}$ respectivas categorias de toxidez (expressa em números absolutos (n) e percentagens)

\begin{tabular}{c|c|c|l}
\hline Níveis & $\mathrm{n}$ & $\%$ & Categoria de toxidez \\
\hline $0,0-0,05$ & 0 & 0,00 & Baixa ou Negativa \\
$0,05-0,1$ & 0 & 0,00 & Média \\
$0,1-1,0$ & 12 & 10,35 & Alta \\
\hline $1,0-2,5$ & 28 & 24,14 & \\
$2,5-5,0$ & 40 & 34,48 & \\
$5,0-10,0$ & 31 & 26,72 & Muito alta \\
$10,0-20,0$ & 5 & 4,31 & \\
\hline TOTAL & 116 & 100,00 & - \\
\hline
\end{tabular}

Quadro 12 - Distribuição do número de incidências das aflatoxinas $G$, por níveis (expressa em números absolutos (n) e percentagens)

\begin{tabular}{|c|c|c|c|c|}
\hline \multicolumn{3}{|c|}{ Niveis } & \multirow{2}{*}{$\frac{n}{1}$} & \multirow{2}{*}{$\frac{\%}{0,86}$} \\
\hline 0,00 & - & 0,075 & & \\
\hline 0,075 & - & 0,75 & 82 & 70,70 \\
\hline 0,75 & - & 1,87 & 16 & 13,79 \\
\hline 1,87 & - & 3,75 & 16 & 13,79 \\
\hline 3,75 & - & 7,50 & 1 & 0,86 \\
\hline 7,50 & & 15,00 & 0 & 0,00 \\
\hline \multicolumn{3}{|c|}{ TOT A L } & 116 & 100,00 \\
\hline
\end{tabular}


Quadro 13 - Distribuição do número de incidências das aflatoxinas B, por níveis e por safras

\begin{tabular}{cccccc}
\hline & \multirow{2}{*}{ Nive1 } & \multicolumn{2}{c}{ "Águas" } & \multicolumn{3}{c}{ "Secas" } \\
\cline { 2 - 6 } & & $\mathrm{n}$ & $\%$ & $\mathrm{n}$ & $\%$ \\
\hline $0,0-0,05$ & 0 & 0,00 & 0 & 0,00 \\
$0,05-0,1$ & 0 & 0,00 & 0 & 0,00 \\
$0,1-1,0$ & 0 & 0,00 & 12 & 25,00 \\
$1,0-$ & 4,5 & 5,88 & 24 & 50,00 \\
$2,5-5,0$ & 30 & 42,65 & 11 & 22,92 \\
$5,0-10,0$ & 5 & 44,12 & 1 & 2,08 \\
$10,0-20,0$ & & 7,35 & 0 & 0,0 \\
\hline T O T A L & 68 & 100,00 & 48 & 100,00 \\
\hline
\end{tabular}

Quadro 14 - Distribuição do número de incidências das aflatoxinas G, por níveis e por safras

\begin{tabular}{lrrrrr}
\hline & & \multicolumn{2}{c}{ “Águas” } & \multicolumn{2}{c}{ “Secas" } \\
\cline { 2 - 6 } & & $\mathrm{n}$ & $\%$ & $\mathrm{n}$ & $\%$ \\
\hline $0,00-$ & 0,075 & 1 & 1,47 & 0 & 0,00 \\
$0,075-$ & 0,75 & 41 & 60,29 & 41 & 85,42 \\
$0,75-$ & 1,87 & 10 & 14,71 & 6 & 12,50 \\
$1,87-$ & 3,75 & 15 & 22,06 & 1 & 2,08 \\
$3,75-$ & 7,50 & 1 & 1,47 & 0 & 0,00 \\
$7,50-$ & 15,00 & 0 & 0,00 & 0 & 0,00 \\
\hline T O T A L & 68 & 100,00 & 48 & 100,00 \\
\hline
\end{tabular}


Quadro 15 - Médias dos valores encontrados para as aflatoxinas B e G, por safra e geral (expressas em partes por milhão)

\begin{tabular}{cccc}
\hline Aflatoxinas & "Águas" & "Seca" & Geral \\
\hline B & 5,50 & 1,76 & 3,24 \\
G & 0,92 & 0,53 & 0,72 \\
\hline
\end{tabular}

